\title{
A Haptic Interface with Motor/Brake System for Colonoscopy Simulation
}

\author{
Evren Samur* Lionel Flaction ${ }^{\dagger} \quad$ Ulrich Spaelter Hannes Bleuler \\ Laboratory of Robotic Systems, Ecole Polytechnique Fédérale de Lausanne (EPFL), Switzerland \\ David Hellier ${ }^{\ddagger}$ Sebastien Ourselin \\ BioMedIA Lab, CSIRO E-Health Research Centre, Brisbane, Australia
}

\begin{abstract}
Training for colonoscopy, which is the examination and treatment of the colon, is often performed on real patients once the physician has passed the novice level. This increases the risk to the patient and lengthens the procedure time. Since a decade ago, there has been research on virtual reality surgery simulators with haptic feedback. The main goal is to provide an alternative to traditional training methods on animals, cadavers or real patients. Haptic feedback is a key feature for every surgery simulator for the training of hand-eye coordination. In this paper, a compact and portable haptic interface is presented for the colonoscopy. The haptic interface provides position data acquisition and force feedback in linear and rotational directions with combined electrical motors and passive brakes to cover a large range of forces. The motorized drives are used for active force feedback and friction compensation and the brakes are used for high force rendering without slipping. This novel design allows decoupled motion in both directions.
\end{abstract}

Index Terms: B.0 [Hardware]: General-Surgery Simulators; I.6.3 [Computing Methodologies]: Simulation and ModelingApplications

\section{INTRODUCTION}

Colonoscopy is a minimally invasive surgery (MIS) procedure where the colon of the patient is examined with an endoscope. The instrument is called a colonoscope which is a long flexible tube with an integrated imaging device at its tip. The colonoscope allows physicians to treat lesions while visualizing the colon. Due to difficult hand-eye coordination, complicated guidance of the flexible endoscope and the risk of colon injury, these techniques need to be performed by highly trained and experienced physicians. The importance of training colonoscopy procedures rises with the growth of variety of colon diseases and growing need to screen as a preventative measure. Computer-based training of this procedure with virtual reality (VR) visualization and haptic feedback offers flexible and repeatable scenarios without risk to the patient. Furthermore, it allows session recording and has therefore distinct advantages over traditional training methods on animals, cadavers or real patients.

Recently a few studies $[4,7,9,5]$ have focused on simulation modeling and visualization of colonoscopy with haptic feedback. Some of these studies $[6,2]$ have already lead to products. Ikuta et. al [4] designed a portable virtual endoscope system which is actuated by several friction rollers and a rubber ball. Although this mechanism provided compact solution, forces in both directions were insufficient for a realistic simulation. Korner and Manner [7] implemented a haptic interface for flexible endoscopy. They attached the tip of the endoscope to a carriage connected to a toothed belt drive actuated by a motor. This implementation required modification on the real endoscope and rotational force feedback was relatively weak. Yi et. al [9], proposed a 2-DOF haptic device which has decoupled translational and rotational force feedback. A

*evren.samur@epfl.ch

$\dagger$ lionel.flaction@epfl.ch

†david.hellier@csiro.au

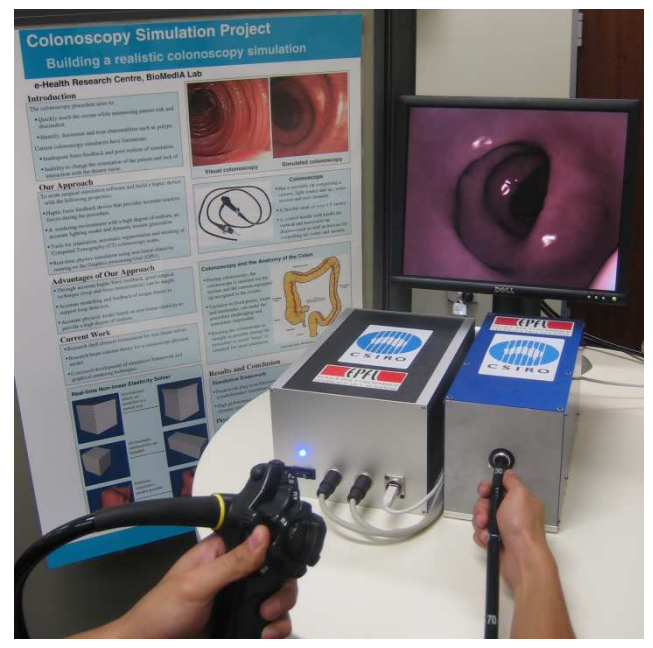

Figure 1: The colonoscopy haptic device is highly portable and rugged. Shown with an Olympus CF-160 colonoscope inserted, and the MILX ${ }^{\text {TM }}$ GastroSim framework rendering a colon model extracted from CT colonography [3]

wire-driven mechanism for the translation and a timing belt mechanism for the rotation were used to decouple the two motions. Nevertheless, since the tip of the colonoscope tube was attached to the device, tool insertion and removal were not possible. The Endoscopy AccuTouch System ${ }^{\circledR}$ [6] is a commercial system for teaching and assessing motor skills for endoscopic procedures including colonoscopy. Using real-time computer graphics, force is transmitted through the flexible scope to provide tactile sensations. However, linear forces are weak and rotational force feedback is neglected in this simulator. The GI Mentor ${ }^{\mathrm{TM}}$ [2] and GI Mentor $\mathrm{II}^{\mathrm{TM}}[8]$ are two commercial simulators that provide hands-on training in both diagnostic and therapeutic endoscopic procedures. These computer-based simulators provide higher force feedback by a pneumatic balloon breaking system, yet the translational and rotational force feedback are not decoupled.

The first prototype realized in our laboratory by Ilic et. al [5] had a differential drive to decouple translation and rotation and a high friction belt to ensure the required forces and the absence of slippage. Although the completed prototype was able to render high forces, compactness and portability requirements led us to consider a new device. To address the need for higher fidelity and complexity in a colonoscopy simulator, we have designed a new haptic interface in order to integrate it with the software simulation framework for colonoscopy (MILX ${ }^{\mathrm{TM}}$ GastroSim) [3]. This paper presents the haptic hardware interface of the training system for the colonoscopy. The haptic interface provides position data acquisition and force feedback in linear and rotational directions with combined electrical motors and passive brakes. The VR environment allows principals of colonoscopy intervention including realistic visualization as well as force rendering for haptic feedback. 


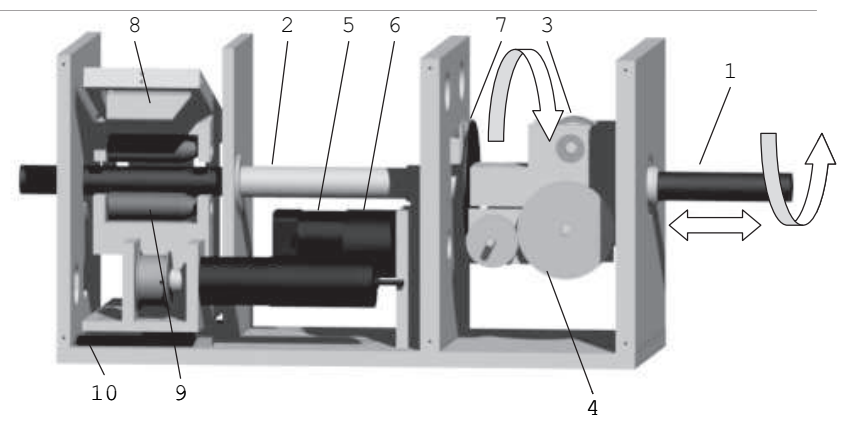

Figure 2: Mechanical design of the haptic interface:(1) Colonoscope, (2) Guidance, (3) Friction rollers, (4) Gears, (5) Motor, (6) Rotational brake, (7) Slip-ring, (8) Linear brake, (9) Cylinders, (10) Linear rails

\section{DESIGN AND PROTOTYPE}

During a typical colonoscopy procedure, the colonoscope is inserted and rotated along the colon. Therefore, the linear and rotational workspace of a simulator should be practically unlimited for a realistic application. In addition, tool re-insertion is also required. Estimated forces and torques for a procedure performed with an adult size colonoscope are in the range of about $\pm 44 \mathrm{~N}$ and $\pm 1 \mathrm{Nm}$ respectively [1].

Considering these requirements, the haptic device is designed to work with an adapted Olympus CF-140 or CF-160 variable stiffness colonoscope. It provides position data acquisition and force feedback in linear and rotational directions. As illustrated in Figure 2, the colonoscope (Fig. 2.1) is inserted in the simulator through a transversal guiding tube (Fig. 2.2). The tube contains windows to allow the contact between the colonoscope and a pair of friction rollers (Fig. 2.3). The rollers are used to track the linear displacement of the colonoscope and to impose linear force feedback, but they also ensure the contact for the rotational movement of the colonoscope. The rollers are connected to a DC motor (not represented in this figure) through a series of gears (Fig. 2.4). The rotational part is fixed on bearings and the electrical connection is provided by a slip-ring pair (Fig. 2.7) that allow an infinite rotational movement of the colonoscope. This rotational movement is also linked to a DC motor (Fig. 2.5) by means of gears. The haptic device includes DC motors for active force feedback and friction compensation as well as brakes for high force rendering during linear displacement and rotation. Maximum rotational torque provided by the DC motor is $0.5 \mathrm{Nm}$, while a powder brake (Fig. 2.6) is used above that value. A mechanical brake (Fig. 2.8) with 2 DOF is designed to be used above $25 \mathrm{~N}$ which is the maximum force that can be exerted by the motor in linear direction. This case could happen, for example, if the operator is pushing the colonoscope against a colon's virtual wall. In this case, the mechanical brake would be closed. The brake is released as soon as the colonoscope is moving back. To make it possible, the brake system is fixed on rails (Fig. 2.10) with linear displacement of $\pm 5 \mathrm{~mm}$ and is constrained by springs of different stiffnesses. This solution is very close to natural behavior. The mechanical brake contains four cylinders (Fig. 2.9) fixed on bearings. When the brake is closed, all the cylinders compress the colonoscope, stopping the linear displacement, but still allowing rotational movement of the colonoscope. Two optical position encoders for linear and rotational displacements are tracked individually by USB devices. The haptic device is interfaced to a PC running Linux 2.6.20 with RTAI patches via USB, allowing transmission at haptic rates with a plug-and-play solution.

Figure 3 shows the first realization of the prototype. A colonoscope, an electronics rack and a foot-pedal are connected to the device as shown in Figure 1.

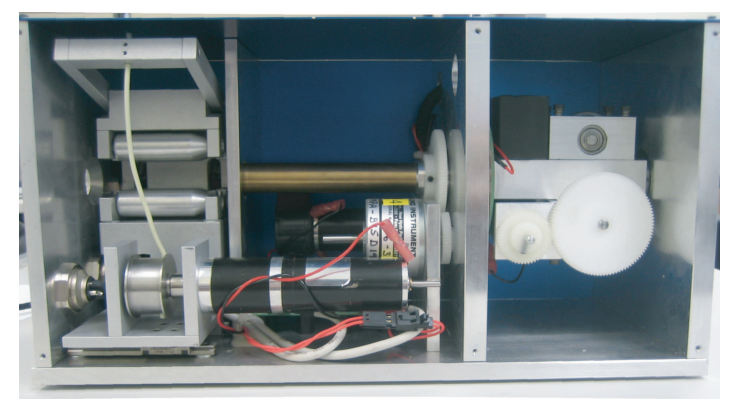

Figure 3: Haptic interface prototype with motor/brake system

\section{CONCLUSIONS AND FUTURE WORK}

In this paper, a compact and portable 2-DOF haptic interface, which provides force feedback in linear and rotational directions, is presented for colonoscopy. As the required maximum forces in a typical colonoscopy intervention vary widely it has been proposed to combine electrical motors and passive brakes to cover a large range of required forces. DC motors are used to simulate the natural friction of the colonoscope when inserting through the colon. A powder brake and a mechanical brake are used if forces or torques applied on the colonoscope are too strong to be maintained by the motors. This novel design allows motion in one direction while impeding the other. The interface has an unlimited linear and rotational workspace and the tool insertion and removal are also possible. In the future, sensors will be integrated into the colonoscope in order to track the device functionalities (e.g., tip bending and inflow/outflow valves) for the virtual reality.

\section{ACKNOWLEDGEMENTS}

This is a joint paper between EPFL and CSIRO, funded by the CSIRO Preventative Health Flagship. We acknowledge the support of the NCCR Co-Me of the Swiss National Science Foundation for the haptic device development.

\section{REFERENCES}

[1] M. N. Appleyard, C. A. Mosse, T. N. Mills, G. D. Bell, F. D. Castillo, and C. P. Swain. The measurement of forces exerted during colonoscopy. Gastrointest. Endosc., 52(2):237-240, 2000.

[2] S. Bar-Meir. A new endoscopic simulator. Endoscopy, 32(11):898-900, 2000.

[3] D. Hellier, E. Samur, J. Passenger, U. Spaelter, H. Frimmel, M. Appleyard, H. Bleuler, and S. Ourselin. A modular simulation framework for colonoscopy using a new haptic device. In Proc. of the 16th Medicine Meets Virtual Reality Conference (MMVR), In Press, 2008.

[4] K. Ikuta, K. Iritani, J. Fukuyama, and M. Takeichi. Portable virtual endoscope system with force and visual display. In Proc. of the IEEE/RSJ International Conference on Intelligent Robots and Systems (IROS), volume 1, pages 720-726, 2000.

[5] D. Ilic, T. Moix, N. M. Cullough, L. Duratti, I. Vecerina, and H. Bleuler. Real-time haptic interface for VR colonoscopy simulation. In Proc. of the 13th Medicine Meets Virtual Reality Conference (MMVR), volume 111, pages 208-212, 2005.

[6] Immersion Medical, Gaithersburg, MD, USA. Endoscopy AccuTouch System ${ }^{\circledR}$. http://www.immersion.com/medical/products/endoscopy/.

[7] O. Korner and R. Manner. Implementation of a haptic interface for a virtual reality simulator for flexible endoscopy. In Proc. of the 11th Symposium on Haptic Interfaces for Virtual Environment and Teleoperator Systems, pages 278-284, 2003.

[8] Simbionix USA Corp., Cleveland, OH. GI Mentor II $^{\mathrm{TM}}$ http://www.simbionix.com/.

[9] S. Y. Yi, H. S. Woo, W. J. Ahn, J. Y. Kwon, and D. Y. Lee. New colonoscopy simulator with improved haptic fidelity. Journal Advanced Robotics, 20(3):349-365, March 2006. 